\title{
A confusion-limited spectral-line survey of Sgr B2(N) at 1, 2, and 3mm: Establishing the organic inventory in molecular clouds
}

\author{
DeWayne T. Halfen and Lucy M. Ziurys \\ Depts. of Chemistry and Astronomy, Arizona Radio Observatory, Laplace Center for \\ Astrobiology, and Steward Observatory, University of Arizona \\ 933 N. Cherry Ave. Tucson, AZ 85721 USA \\ email: halfendt@as.arizona.edu
}

\begin{abstract}
We present preliminary results of an spectral-line survey at 1, 2, and $3 \mathrm{~mm}$ of the galactic center cloud Sgr B2(N). With the current data, several simple prebiotic molecules have been conclusively identified, while several more complex molecules have not. When complete, this survey will provide an accurate database of the gas-phase organic inventory in Sgr B2(N).
\end{abstract}

Keywords. ISM: molecules, astrochemistry, astrobiology, submillimeter, ISM: clouds

\section{Introduction}

Life on Earth is postulated to have started from simple chemical compounds. Where did this organic starting material come from? It is possible that the organic matter on the early Earth was delivered by comets, meteorites, and interplanetary dust particles. A diverse array of organic compounds have been found in meteorites, including sugars and amino acids (Cooper et al. 2001, Pizzarello et al. 2001). The most likely source of the meteoritic organic compounds is the interstellar medium (ISM). In the ISM, large quantities of organic matter have been found in giant molecular clouds. About half of the 140 molecules found in space have been detected in the Galactic center cloud Sgr B2(N), including the most complex organic species. Hence, the overall chemical composition of this source is of great interest. Therefore, we are conducting a spectral-line survey of this source to determine the identify and abundance of each molecule.

\section{Observations}

Observations towards Sgr B2(N) cover the millimeter atmospheric windows at 3 (65$116 \mathrm{GHz}), 2(130-180 \mathrm{GHz})$, and 1 (210-280 GHz) $\mathrm{mm}$ in wavelength. The observations were performed at the two radio telescopes of the Arizona Radio Observatory, the $12 \mathrm{~m}$ telescope on Kitt Peak and the Submillimeter Telescope (SMT) on Mt. Graham. The $12 \mathrm{~m}$ operates at 2 and $3 \mathrm{~mm}$ and the SMT performs measurements at $1 \mathrm{~mm}$. The temperature scale is given as $T_{\mathrm{R}}^{*}$ for the data from $65-180 \mathrm{GHz}$, and $T_{\mathrm{A}}^{*}$ from $210-280 \mathrm{GHz}$. Spectral coverage is currently $78 \%, 66 \%$, and $40 \%$ at 3,2 , and $1 \mathrm{~mm}$, respectively, see Figure 1 . This survey is conducted to the confusion limit in SSB mode, where the spectrum consists of a continuum of spectral lines, and maximum information can be obtained.

\section{Results}

One molecule of great interest is glycolaldehyde, $\mathrm{CH}_{2} \mathrm{OHCHO}$. The previous identification of this species has based on six transitions, five of which were contaminated by other molecules (Hollis et al. 2000). In the current data set, 40 transitions of glycolaldehyde were measured. Emission was present at all transitions, eight of which were uncontaminated. There were no "missing" lines. Therefore, glycolaldehyde is unambiguously present 

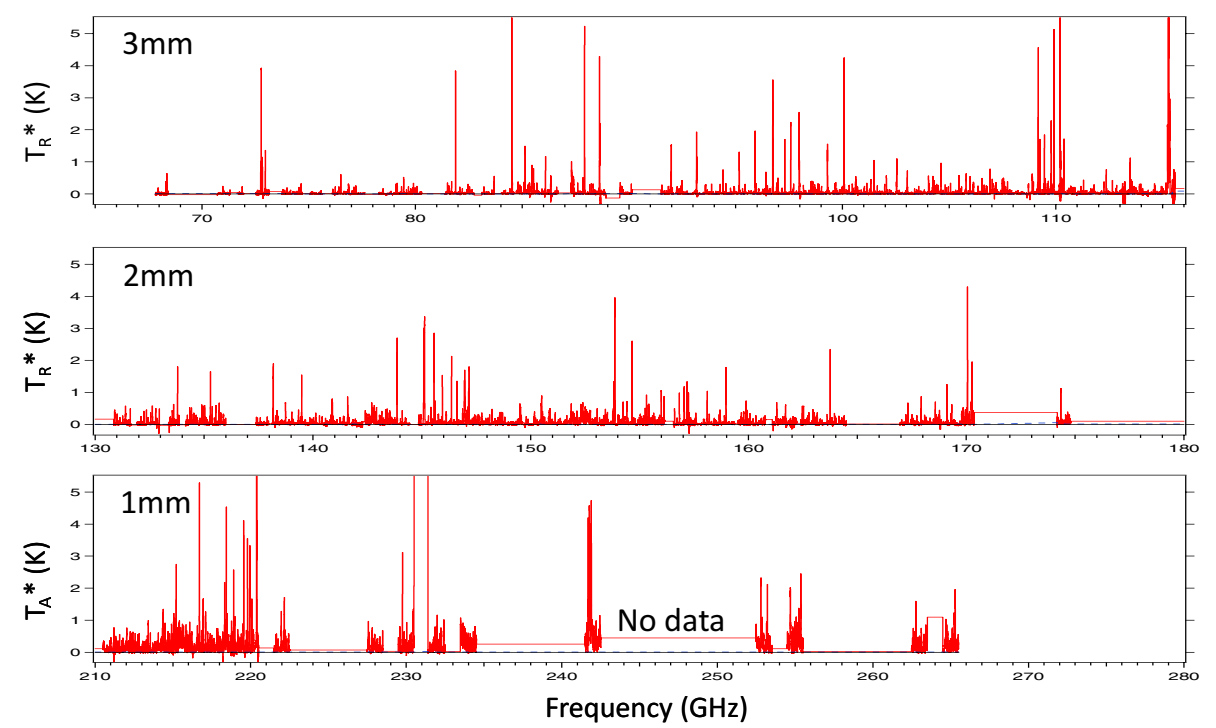

Figure 1. Current survey coverage of Sgr B2(N)

in Sgr B2(N), with an abundance of $2 \times 10^{-11}$ (Halfen et al. 2006). Another compound of interest is formamide, $\mathrm{NH}_{2} \mathrm{CHO}$, the smallest species to contain a peptide bond, i. e., the important linkage between amino acids in proteins. Over 60 lines of formamide were observed, and these data give an abundance wrt $\mathrm{H}_{2}$ of $1 \times 10^{-10}$. The next most complex simple protein analog is acetamide, $\mathrm{CH}_{3} \mathrm{CONH}_{2}$. For this molecule, 335 transitions are present in our current data, and emission is detected at all of these frequencies. Acetamide is present in Sgr B2(N) with an abundance of $1 \times 10^{-10}$. The simplest molecule with an amine group is methyl amine. Over 220 transitions of this compound have been detected in our survey, yielding an abundance in Sgr B2(N) of $1 \times 10^{-9}$ wrt $\mathrm{H}_{2}$. We also searched for ethyl amine. However, there are 20 transitions of this molecule where no emission is detected, and therefore, it is not present in $\mathrm{Sgr} B 2(\mathrm{~N})$ with an upper limit wrt $\mathrm{H}_{2}$ of $<3 \times 10^{-11}$ (Apponi et al. 2008). Several other complex molecules were not apparent in our survey. The 3-carbon sugar dihydroxyacetone (DHA), $\mathrm{HOCH}_{2} \mathrm{COCH}_{2} \mathrm{OH}$, has been claimed to be present in Sgr B2(N) by Widicus et al. (2005). However, in our survey, we have 20 transitions where there is no emission (Apponi et al. 2006a). In addition, our data indicate that the sugar-like molecule hydroxyacetone, $\mathrm{CH}_{3} \mathrm{COCH}_{2} \mathrm{OH}$, does not exist in Sgr B2(N) with an upper limit of $2 \times 10^{-12}$ (Apponi et al. 2006b).

This research is supported by NASA through the Astrobiology Institute under Cooperative Agreement No. CAN-02 OSS02 and by an NSF grant AST-0602282.

\section{References}

Apponi, A. J., Halfen, D. T., Ziurys, L. M., Hollis, J. M., Remijan, A. J., \& Lovas, F. J. 2006a, ApJ (Letters), 634, L29

Apponi, A. J., Hoy, J. J., Halfen, D. T., Ziurys, L. M., \& Brewster, M. A. 2006b, ApJ, 652, 1787 Apponi, A. J., Sun, M., Halfen, D. T., Ziurys, L. M., \& Müller, H. S. P. 2008, ApJ, 673, 1240

Cooper, G., Kimmich, N., Belisle, W., Sarinana, J., Brabham, K., \& Garrel, L. 2001, Nature, 414,879

Halfen, D. T., Apponi, A. J., Woolf, N. J., Polt, R., \& Ziurys, L. M. 2006, ApJ, 639, 237

Hollis, J. M, Lovas, F. J., \& Jewell, P. R. 2000, ApJ (Letters), 540, L107

Pizzarello, S., Huang, Y., Becker, L., Poreda, R. J., Nieman, R. A., Cooper, G., \& Williams, M. 2001, Science, 293, 2236

Widicus Weaver, S. L. \& Blake, G. A. 2005, ApJ (Letters), 624, L33 